\title{
Attempting a Change in Human Behavior in ICU in COVID Era: Handle with Care!
}

\author{
Anuj M Clerk
}

Indian Journal of Critical Care Medicine (2020): 10.5005/jp-journals-10071-23539

Coronavirus can spread by contact, droplet, and air-borne transmission. Thousands of healthcare workers are infected, and many have lost lives due to COVID-19 infection, which brings infection control at center stage. Compliance with hand hygiene measures (hand wash and handrub application), although adequately emphasized, remains suboptimal. This leads to contamination of hands or gloves on the hands to harbor organisms, including coronaviruses, and carried to face near to oronasal orifices while touching face with hands, resulting in infection in healthcare workers.

Kwok et al. ${ }^{1}$ at the University of New South Wales studied 26 medical students on Video footages. They observed students touching face as many as 23 times in an hour of which $44 \%$ times involved mucous membranes of mouth, nose, and eyes. This behavior can lead to direct inoculation of coronaviruses from contaminated hands on to the mucous membranes. Ten persons studied for 3 hours doing office work touched their mucous membranes on face 15.7 times per hour. ${ }^{2}$ Thus, one touches face more often than washing hands making contaminated hands potential transport mechanisms. Touching face is human behavior that differs in extent in different people, in different surroundings, and subject to change with mood.

Wearing face mask reduced face touching from 20 times an hour to 5.4 times an hour in hemato-oncology staff in one study, ${ }^{3}$ but loose mask slipping down the nose attracts more hand contacts, on the contrary. Bringing change with habit reversal training (HRT) methods consisting of three elements, (1) awareness training, (2) competing response training, and (3) social support training (for children mainly), is deemed highly effective in 35 of 39 studies included in a recently published review seems promising. ${ }^{4}$ Use of camera on head gear, CCTV monitoring and real-time call back from monitor, use of full face shield, and use of Powered air purifier Respirator (PAPR) with hood covering face are other alternatives one can think of preventing hand touching face, but cost may prohibit its applicability.

In this issue of IJCCM, Senthilkumaran et al. attempted use of adhesive tapes applied across the elbow to serve as reminders when one tries to flex them for touching the face. ${ }^{5}$ This tapes are intended to cause discomfort while bending elbow more than 90 degrees (as used while touching face) and thereby provide reminder not to touch the face. While authors must be appreciated for their innovative method to bring behavioral change, repeated application of tapes for more than few days seems unlikely. Absence of face touching in the first 3 days may be due to "Hawthorne effect," as candidates knew that they are being observed rather than effect of tapes itself. As there was no behavioral training involved, three of nine volunteers started touching face after 3 days, despite tapes on their elbows,
Department of Intensive Care, Sunshine Global Hospital, Surat, Gujarat, India

Corresponding Author: Anuj M Clerk, Department of Intensive Care, Sunshine Global Hospital, Surat, Gujarat, India, Phone: +91 7574851424, e-mail: anujmclerk@hotmail.com

How to cite this article: Clerk AM. Attempting a Change in Human Behavior in ICU in COVID Era: Handle with Care! Indian J Crit Care Med 2020;24(8):626-627.

Source of support: Nil

Conflict of interest: None

which questions sustainability of such a measure. Physical injuries due to such taping like traction blisters, adhesive material reaction, infections under tapes, skin peals, stigma involved in application of tapes (akin to branding) and hindrance in use of hand in other functions due to tapes, is likely to prohibit prolonged use, limit its wider applicability, and acceptance by ICU staff outside study period.

The Transtheoretical Model, known as "The stages of Change Model", was developed by Prochaska and DiClemente in $1983^{6}$ and is worth revisiting at this time when one aims at bringing an enduring change in human behavior. The methods we adopt must be practicable on long-term and acceptable to the group of individuals on which we want to bring the change. As stated by the inventors, most difficult is maintenance stage, i.e., the change should stand the test of time, and for that the solution (action) we bring has to be durable, practical, acceptable, and void of any unwanted effects to individuals (Fig. 1).

Simplified versions of HRT as proposed by Heinicke et al. ${ }^{4}$ in their article if rehershed enough, seems workable, sustainable, and acceptable solution.

\begin{tabular}{ll}
\hline Steps & Simplified habit reversal training \\
\hline 1 & $\begin{array}{l}\text { Identify a competing maneuver that makes touching } \\
\text { face impossible, for example make a fist and bring near } \\
\text { tummy }\end{array}$ \\
2 & $\begin{array}{l}\text { Practice competing maneuver each time one tries to } \\
\text { touch face, interrupting hand to face movement. } \\
\text { Use competing maneuver each time when one gets } \\
\text { urge to touch face }\end{array}$ \\
\hline
\end{tabular}

In COVID era where hospitals are struggling to maintain workforce, introduction of any new rule or regime that can further demotivate already stressed staff working in full PPE kits requires extreme caution. However, "Necessity is mother of invention," and

() The Author(s). 2020 Open Access This article is distributed under the terms of the Creative Commons Attribution 4.0 International License (https://creativecommons. org/licenses/by-nc/4.0/), which permits unrestricted use, distribution, and non-commercial reproduction in any medium, provided you give appropriate credit to the original author(s) and the source, provide a link to the Creative Commons license, and indicate if changes were made. The Creative Commons Public Domain Dedication waiver (http://creativecommons.org/publicdomain/zero/1.0/) applies to the data made available in this article, unless otherwise stated. 


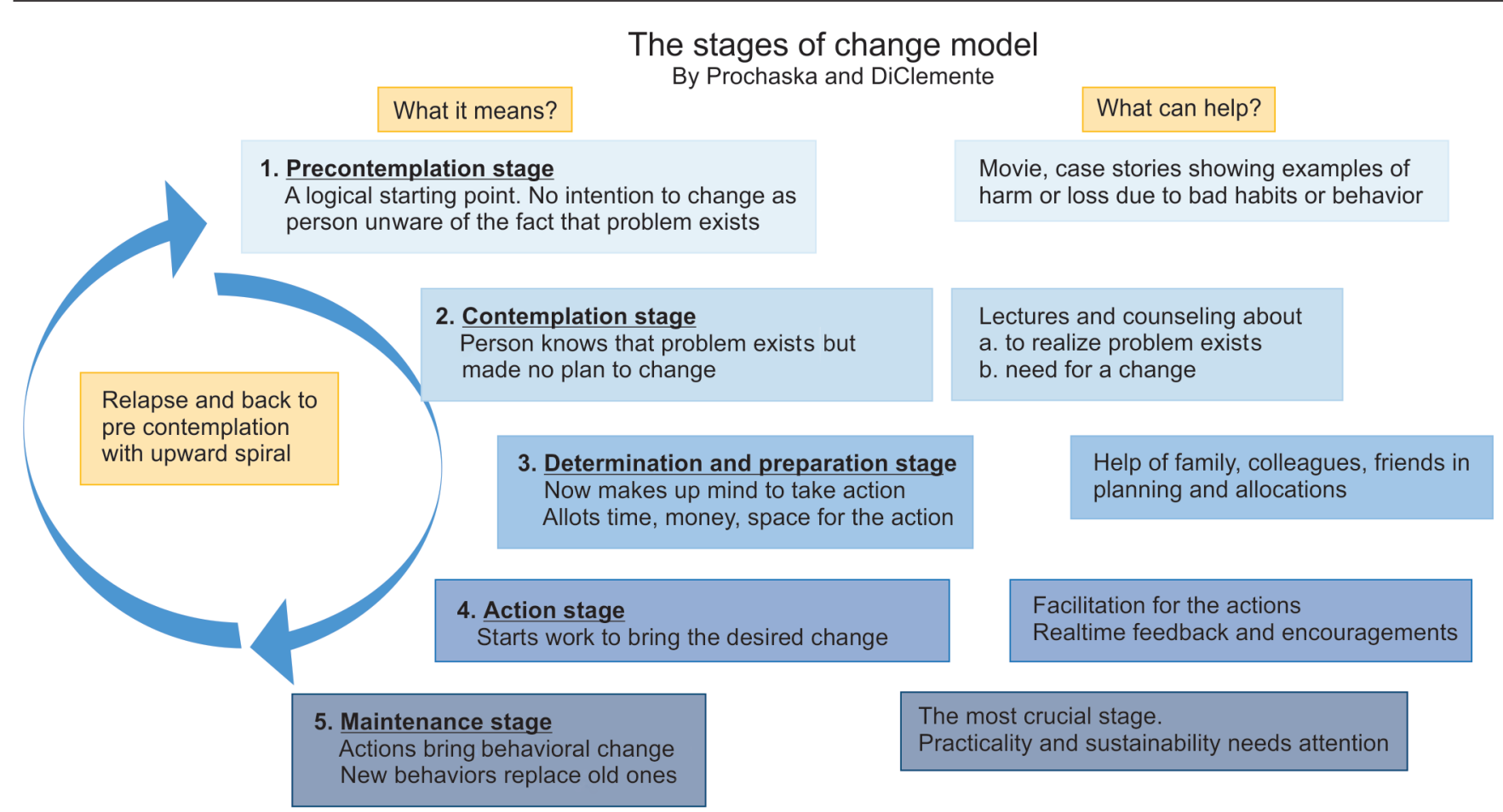

Fig. 1: Stages of change model

COVID pandemic ${ }^{6}$ has brought us unprecedented adversity (or say opportunity) to work on solutions for reducing hand-to-face touch behaviors. Solution if invented under the pressure of preventing spread of coronavirus, and integrated in routine practice, will have benefits lasting beyond corona pandemic in safeguarding healthcare workers handling infectious patients.

In the end, one must welcome the innovative gestures of authors to look for solutions to bring reduction in touching face, behavioral change by established principles of human psychology, and behavioral training (like HRT or simplified HRT) may be more enduring and encouraging. Thus, attempting a change in human behavior in ICU in COVID era needs caution and should bear a tag, "Handle with Care"...!!

\section{References}

1. Kwok YL, Gralton J, McLaws ML. Face touching: a frequent habit that has implications for hand hygiene. Am J Infect Contr 2015;43(2): 112-114. DOI: 10.1016/j.ajic.2014.10.015.
2. Nicas M, Best D. A study quantifying the hand-to-face contact rate and its potential application to predicting respiratory tract infection. J Occup Environ Hyg 2008;5(6):347-352. DOI: 10.1080/15459620802003896.

3. Lucas TL, Mustain R, Goldsby RE. Frequency of face touching with and without a mask in pediatric hematology/oncology health care professionals. Pediatr Blood Cancer 2020;67(9):e28593. DOI: 10.1002/ pbc.28593.

4. Heinicke MR, Stiede JT, Miltenberger RG, Woods DW. Reducing risky behavior with habit reversal: a review of behavioral strategies to reduce habitual hand-to-head behaviour. J Appl Behav Anal 2020;53(3):1225-1236. DOI: 10.1002/jaba.745.

5. Senthilkumaran S, Arathisenthil SV, Meenakshisundaram R. Not touching the face is harder than it sounds: need for an intervention. Indian J Crit Care Med 2020;24(8):662-663.

6. Prochaska J, DiClemente C. Stages and processes of self-change of smoking: towards an integrated model of change. J Consult Clin Psychol 1983;51(3):390-395. DOI: 10.1037/0022-006X.51. 3.390 . 